\title{
miR-214 promotes osteosarcoma tumor growth and metastasis by decreasing the expression of PTEN
}

\author{
CHUN-JIE LIU ${ }^{1}$, KUN-LUN YU², GUO-LI LIU ${ }^{2}$ and DE-HU TIAN ${ }^{2}$ \\ ${ }^{1}$ Department of Orthopedics, Tangshan Gongren Hospital, Tangshan, Hebei 063000; ${ }^{2}$ Department of Orthopedics, \\ The Third Affiliated Hospital of Hebei Medical University, Shijiazhuang, Hebei 050051, P.R. China
}

Received November 2, 2014; Accepted July 21, 2015

DOI: $10.3892 / \mathrm{mmr} .2015 .4197$

\begin{abstract}
MicroRNAs (miRNAs) are a class of small non-coding RNAs, which function as critical gene regulators by targeting mRNAs for translational repression or degradation, and they are essential in cancer development and progression. Several previous studies have indicated that abnormal expression of miRNAs occurs frequently in human osteosarcoma (OS) tissues compared with that of adjacent normal tissues. In the present study, the role of miR-214 in the progression and metastasis of OS was investigated. The expression of miR-214 was frequently increased in OS tissues and cell lines. Inhibition of miR-214 in OS cell lines markedly suppressed cell proliferation, migration and invasion. Phosphatase and tensin homolog (PTEN) was identified as a direct target of miR-214, and ectopic expression of miR-214 inhibited PTEN by directly binding to its 3 '-untranslated region. The expression of miR-214 negatively correlated with PTEN in OS tissues. Together, these data indicated that miR-214 acts as an oncogenic miRNA and may contribute to the progression, and metastasis of OS, suggesting that miR-214 may be a potential novel diagnostic and therapeutic target of OS.
\end{abstract}

\section{Introduction}

Osteosarcoma (OS) is one of the most common primary malignant bone tumors in children and adults $(1,2)$. It occurs predominantly around regions with active bone growth and repair. Emerging evidence suggests that OS is caused by genetic and epigenetic changes, which interrupt osteoblast differentiation from mesenchymal stem cells (3). Advances in OS therapy over the past decade have improved patient outcomes (4), and the 5-year survival rate of patients with OS has markedly improved.

Correspondence to: Dr De-Hu Tian, Department of Orthopedics, The Third Affiliated Hospital of Hebei Medical University, 139 Ziqiang Road, Shijiazhuang, Hebei 050051, P.R. China

E-mail: tiandehu@hotmail.com

Key words: osteosarcoma, microRNA, cell proliferation, phosphatase and tensin homolog, miR-214
However, the outcome remains poor and the majority of patients succumbed to pulmonary metastases (5).

MicroRNAs (miRNAs), a class of small non-coding RNA molecules, result in translational repression or degradation, and contribute to the inhibition of gene expression $(6,7)$. miRNAs exert a significant role in a wide range of physiological and pathological processes, including tumorigenesis (8). Increasing evidence implicates miRNAs in cancer progression, including tumor growth, differentiation, invasion, metastasis and angiogenesis (9). It has been demonstrated that several miRNAs are dysregulated in OS tissues or cell lines (10-15). For instance, miR-15a and miR-16-1 induce apoptosis and cell cycle arrest in OS by inhibiting the expression of cyclin D1 (16), while miR-20a increases the metastatic potential of OS cells by regulating the expression of Fas (17). The expression of miR-21 was revealed to be significantly upregulated in OS tissues, and miR-21 deficiency markedly reduced the invasion and migration ability of MG-63 cells by negatively regulating RECK (10).

Previous studies have demonstrated that miR-214 can act as a tumor suppressor or an oncogene in different cancer types, which may be dependent on cellular context (18-24). For example, miR-214 as a tumor suppressor may promote cell proliferation in nasopharyngeal carcinoma (25). It was demonstrated that miR-214 regulates gastric cancer cell proliferation, migration and invasion by targeting PTEN (26), and suppresses the growth and invasiveness of cervical cancer cells by targeting UDP-N-acetyl-D-galactosamine:polypeptide $\mathrm{N}$-Acetylgalactosaminyltransferase 7 (27). However, the role of miR-214 in the progression and metastasis of OS remains to be elucidated. Therefore, the present study investigated the expression of miR-214 and its role in OS tissues and cell lines, aiming to demonstrate that miR-214 is a potential therapeutic target for the treatment of OS.

\section{Materials and methods}

Cell culture and tissue samples. A total of 22 paired OS and matched normal non-tumor tissues were obtained from the Department of Orthopedics, Tangshan Gongren Hospital (Tangshan, China). The tissues were immediately stored in liquid nitrogen until use. All samples were derived from patients who had not received adjuvant treatment, including radiotherapy or chemotherapy, prior to surgery. Written 
informed consent was obtained from all patients and this study was approved by the Ethics Committee of Tangshan Gongren Hospital.

Human OS cell lines, U2OS, Saos-2 and MG-63, were obtained from American Type Culture Collection (Rockville, MD, USA) and were cultured in Dulbecco's modified Eagle's medium (DMEM; Hyclone, Beijing, China), RPMI-1640 (Hyclone) and DMEM, respectively, supplemented with $10 \%$ fetal bovine serum (FBS; Hyclone), $100 \mathrm{mg} / \mathrm{ml}$ streptomycin and $100 \mathrm{IU} / \mathrm{ml}$ penicillin, at $37^{\circ} \mathrm{C}$ with $5 \% \mathrm{CO}_{2}$. The human hFOB 1.19 osteoblast cell line was maintained in DMEM/F12 medium (Hyclone), supplemented with 10\% FBS, $100 \mathrm{mg} / \mathrm{ml}$ streptomycin and $100 \mathrm{IU} / \mathrm{ml}$ penicillin (Hyclone) at $37^{\circ} \mathrm{C}$ with $5 \% \mathrm{CO}_{2}$. TargetScan 6.2 software (www.targetscan. $\mathrm{org} /$ ) was used to assess miR-214, then from a number of candidates, PTEN was selected for validation.

RNA isolation and reverse transcription-quantitative polymerase chain reaction (RT-qPCR). The total RNA and miRNA (miR) were isolated using RNeasy Mini and miRNeasy Mini kits (Qiagen, Valencia, CA, USA), according to the manufacturer's instructions. The expression of miR-214 was determined by RT-qPCR, using TaqMan MicroRNA assay kits (ABI, Foster City, CA, USA) on a LightCycler 480 system II (Roche, Basel, Switzerland). The primer sequences were as follows: PTEN, forward: 5'-CCAGGACCAGAGGAA ACCT-3' and reverse: 5'-GCTAGCCTCTGGATTTGA-3'; GAPDH, forward: 5'-ATGTCGTGGAGTCTACTGGC-3' and reverse: 5'-TGACCTTGCCCACAGCCTTG-3'. Primers were purchased from Invitrogen Life Technologies (Shanghai, China). The expression of PTEN was determined using SYGR green real time PCR (Takara Bio, Inc., Tokyo, Japan). The RT-qPCR data were normalized using the $2^{-\Delta \Delta \mathrm{Ct}}$ method relative to GAPDH as described previously (28).

Plasmid construction and cell transfection. Anti-miR-214 (product ID: AM12124), anti-miR negative control (product ID: AM17010), miR-214 (product ID: PM12124) and miR negative control (product ID: AM17110) were purchased from Ambion (Austin, TX, USA). For the luciferase reporter assay, the following primer was used: Forward, 5'-CGAGCT CGGACGAACTGGTGTAATG-3' and reverse, 5'-CGACGC GTGTCCAGAGTCCAGCATAA-3'. The PCR fragment was inserted into a pMir-Report vector (Ambion) between the SacI and $M l u \mathrm{I}$ restriction sites. The mutation was performed using a fast mutation kit (New England Biolabs, Ipswich, Canada), according to the manufacturer's instructions. The transfection was performed when cells were grown to $80 \%$ confluence, using Lipofectamine 3000 (Invitrogen Life Technologies, Carlsbad, CA, USA), according to the manufacturer's instructions. This work was in agreement with the National Health and Family Planning Commission Guidelines.

Luciferase activity assay. The luciferase activity assay was performed, as previously described (29). Briefly, the

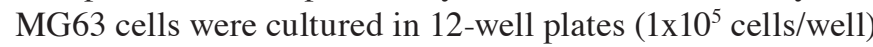
and were cotransfected with wild-type (WT) or mutated (Mut) 3'-untranslated regions (UTRs) of PTEN luciferase reporter constructs, and miR-214 or control mimic using Lipofectamine 3000 . Following incubation for $24 \mathrm{~h}$, the cells were harvested and luciferase activity was determined using a Dual-Luciferase Reporter assay kit (Promega, Madison, WI, USA).

Cell viability assay. The Cell Counting kit-8 (CCK-8) assay was used as a qualitative index of cell viability, which is based on the conversion of a water-soluble tetrazolium salt, 2-(2-methoxy-4-nitrophenyl)-3-(4-nitrophenyl)-5-(2,4disulfophenyl)-2H-tetrazolium, to a water soluble formazan dye upon reduction by dehydrogenases in the presence of an electron carrier (30). The cells were plated into 96-well microplates and subsequently the cell count was performed using CCK-8 (Dojindo Molecular Technologies, Inc., Beijing, China), according to the manufacturer's instructions. Briefly, $10 \mu \mathrm{l}$ CCK-8 solution was added to each well and the samples were incubated for $1 \mathrm{~h}$ prior to the absorbance being measured at $450 \mathrm{~nm}$ using the SpectraMax M5 (Molecular Devices, Sunnyvale, CA, USA).

Colony formation assay. A total of 500 transfected MG- 63 cells were seeded into 6-well plates and maintained in DMEM, containing 10\% FBS for 14 days. The cells were subsequently fixed and stained with $100 \%$ methanol for $20 \mathrm{~min}$, followed by $0.5 \%$ crystal violet for $15 \mathrm{~min}$ at room temperature. Visible colonies were quantified using an inverted microscope (IX73; Olympus, Tokyo, Japan).

In vitro migration and invasion assay. Migration and invasion assays were performed using Transwell chambers. For the migration assay, $5 \times 10^{4}$ cells were seeded into the upper chamber of Transwell plates (BD Bioscience, San Jose, CA, USA). For the invasion assay, $1 \times 10^{5}$ cells were added into the upper chamber precoated with matrigel (BD Bioscience). In each assay, the cells were maintained in medium without serum in the upper chamber and medium, containing $10 \%$ FBS, was added to the lower chamber as a chemoattractant. Following incubation for $24 \mathrm{~h}$, the cells failing to migrate or invade through the membrane were wiped away. The membranes were subsequently fixed and stained with $0.5 \%$ crystal violet. A total of four random fields were counted per chamber using an inverted microscope (Olympus) and each experiment was repeated three times.

Western blotting assay. The cells were harvested and resuspended in phosphate-buffered saline. Following centrifugation at $100 \mathrm{x} \mathrm{g}$ for $5 \mathrm{~min}$ at room temperature, the pellet was lysed in ice-cold Lysis Buffer [50 mM Tris (pH 7.4), $150 \mathrm{mM} \mathrm{NaCl}, 1 \%$ TritonX-100, $1 \%$ sodium deoxycholate, $0.1 \%$ SDS, 2 mM sodium pyrophosphate, $25 \mathrm{mM} \beta$-glycerophosphate, $1 \mathrm{mM}$ EDTA, $1 \mathrm{mM} \mathrm{Na} \mathrm{VO}_{4}, 0.5 \mu \mathrm{g} / \mathrm{ml}$ leupeptin and PMSF], containing $1 \%$ Halt Protease and Phosphatase Inhibitor Cocktail (Thermo Fisher Scientific, Waltham, MA, USA) for $30 \mathrm{~min}$. The supernatant was collected following centrifugation at $4^{\circ} \mathrm{C}$ for $10 \mathrm{~min}$ at $10,142 \mathrm{x} \mathrm{g}$ and the protein concentration was determined using a BCA protein assay kit (Tiangen Biotech Co., Ltd., Beijing, China) with the SpectraMax M5 spectrophotometer at $562 \mathrm{~nm}$. The protein samples were denatured using sample loading buffer (Beyotime Institute of Biotechnology, Haimen, China) for $10 \mathrm{~min}$ at $95^{\circ} \mathrm{C}$ and stored at $4^{\circ} \mathrm{C}$ for future use. Equal concentrations $(1.5-2.0 \mu \mathrm{g} / \mu \mathrm{l})$ of the proteins were 
A

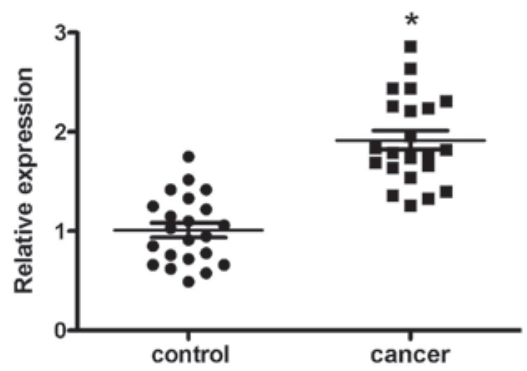

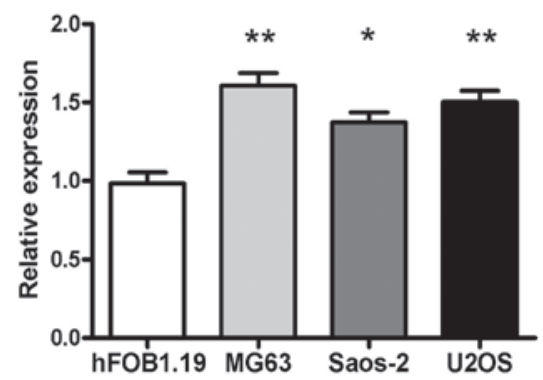

Figure 1. miR-214 was increased in OS tissues and cell lines. (A) The expression of miR-214 was significantly increased in OS tissues compared with that in the corresponding normal tissues. (B) The expression of miR-214 was significantly increased in three OS cell lines, U2OS, Saos-2 and MG-63, compared with the human hFOB1.19 osteoblast cell line $\left({ }^{*} \mathrm{P}<0.05\right.$ and ${ }^{* *} \mathrm{P}<0.01$, compared with the control group). OS, osteosarcoma; miR, microRNA.

A

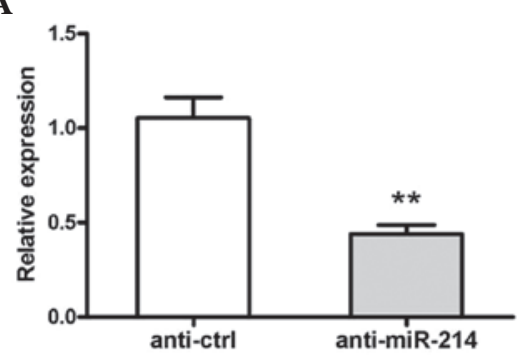

C

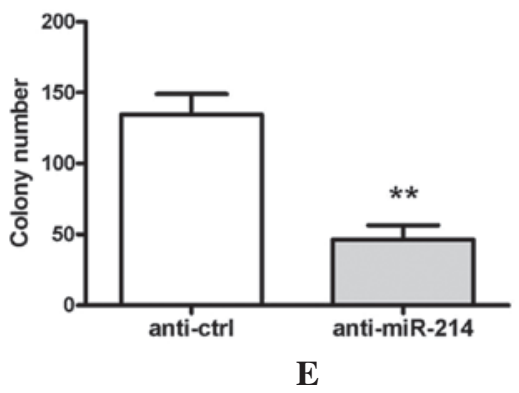

B

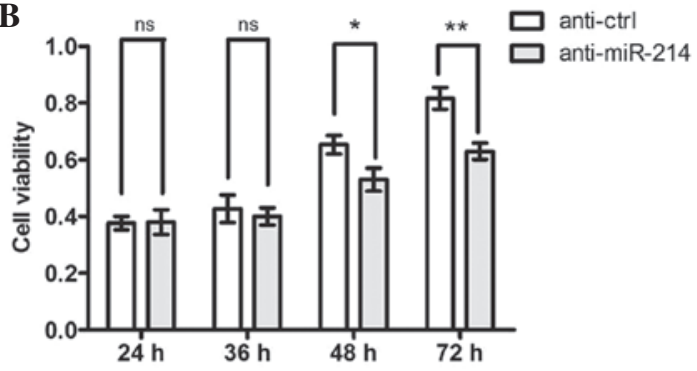

D

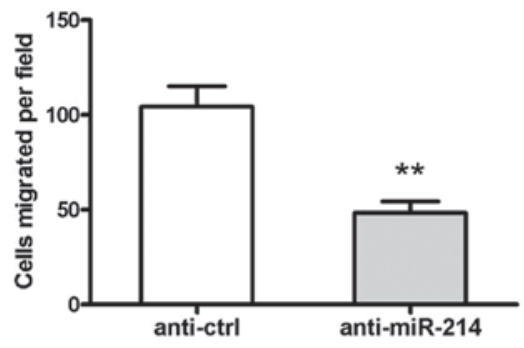

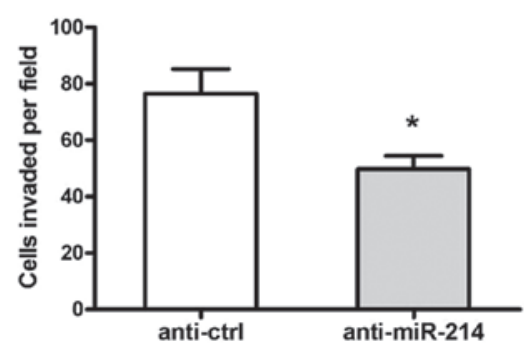

Figure 2. Inhibition of miR-214 suppresses OS cell proliferation and motility. (A) The expression of miR-214 was significantly decreased following transfection of anti-miR-214. (B) An MTT assay was performed to examine the cell proliferation of MG-63 cells. Transfection of anti-miR-214 significantly inhibited MG-63 cell proliferation. (C) Transfection of anti-miR-214 significantly inhibited MG-63 cell colony formation. (D) An in vitro migration assay of MG-63 cells transfected with anti-miR-214 or anti-miR-NC. Transfection of anti-miR-214 significantly suppressed MG-63 cell migration. (E) An in vitro invasion assay of MG-63 cells transfected with anti-miR-214 or anti-miR-NC. Transfection of anti-miR-214 significantly suppressed MG-63 cell invasion. ( $\mathrm{P}<0.05$ and ${ }^{* *} \mathrm{P}<0.01$, compared with the control group). OS, osteosarcoma; miR, microRNA.

separated on $10 \%$ SDS-PAGE gels (Beyotime Institute of Biotechnology) and transferred onto a polyvinylidene fluoride membrane (EMD Millipore, Billerica, MA, USA). The membrane was blocked with 5\% non-fat milk in Tris-buffered saline with 20\% Tween-20 (TBST) for $1 \mathrm{~h}$ at room temperature, and was subsequently incubated with primary antibodies at $4^{\circ} \mathrm{C}$ overnight. The membrane was incubated with the appropriate horseradish peroxidase (HRP)-conjugated secondary antibody, at a dilution of 1:10,000 in blocking buffer, for $1 \mathrm{~h}$ at room temperature. The protein bands were observed using enhanced chemiluminescence (HRP substrate; EMD Millipore) and exposed in a dark room. All antibodies were diluted in 5\% skimmed milk in TBST. The antibodies against PTEN (monoclonal, rabbit anti-human, 


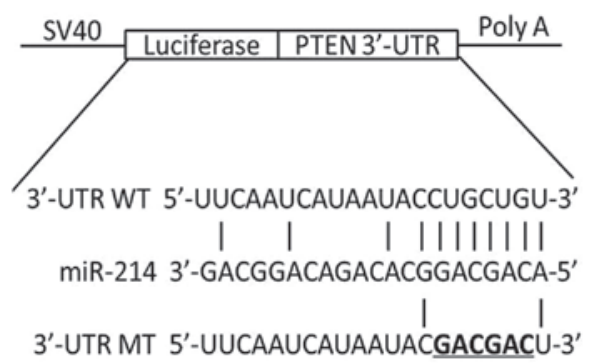

C

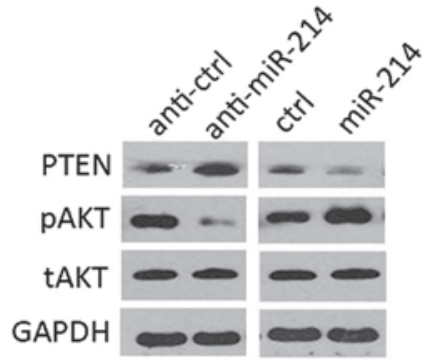

B

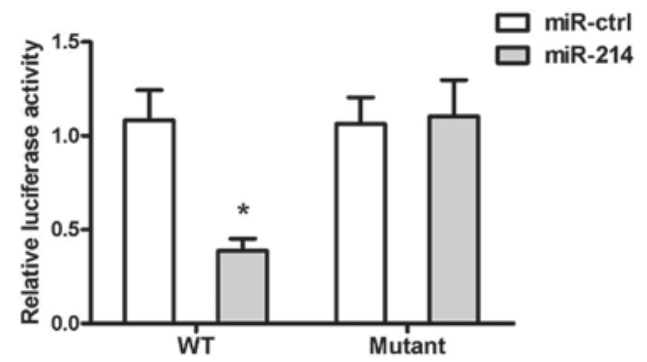

D

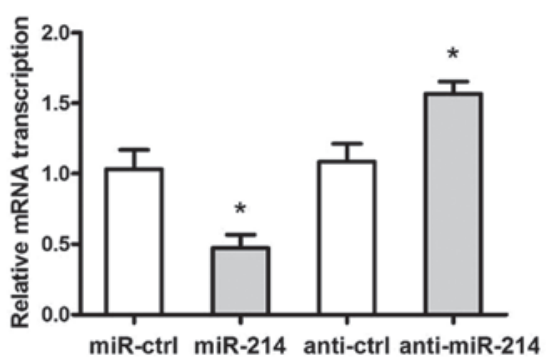

Figure 3. PTEN is a direct target of miR-214. (A) Computational analysis revealed that miR-214 potentially targeted PTEN. (B) HEK293 cells were cotransfected with miR-214 and WT or Mut 3'-UTR luciferase reporter construct. (C) The protein expression levels of PTEN, pAKT, tAKT and GAPDH were detected by western blotting in MG-63 cells transfected with miR-214/ctrl or anti-miR-214/anti-ctrl. (D) The protein expression of PTEN was quantified using Quality One software. ("P<0.05, compared with the control group). miR, microRNA; UTR, untranslated region, WT, wild-type; Mut, mutant; p, phosphorylated; t, total; ctrl, control; PTEN, phosphatase and tensin homolog.

A

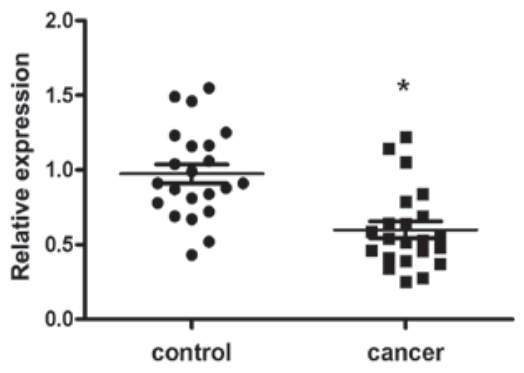

B

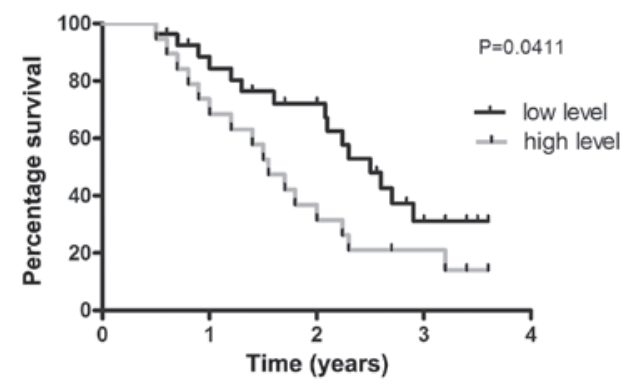

Figure 4. miR-214 negatively correlates with PTEN in OS tissues. (A) The mRNA expression of PTEN was determined by reverse transcription-quantitative polymerase chain reaction and was markedly decreased in OS tissues. (B) Kaplan-Meier survival curves were generated for 35 OS cases. Low expression of miR-214 (black) was defined as long survival, and high expression (light grey) was defined as short survival ("P<0.05, compared with the control group). OS, osteosarcoma; miR, microRNA; PTEN, phosphatase and tensin homolog.

cat.no. ab32199, 1:1,000),pAkt (monoclonal, rabbit anti-human, cat. no. ab81283, 1:7,000), Akt (monoclonal, rabbit anti-human, cat. no. ab32505, 1:5,000), GAPDH (monoclonal, rabbit anti-human, cat. no. ab181602, 1:10,000), $\beta$-actin (monoclonal, rabbit anti-human, cat. no. ab6276, 1:10,000), HRP-secondary antibody (goat anti-rabbit, cat. no. ab6721, 1:10,000), were purchased from Abcam (Cambridge, UK). The protein expression level of PTEN protein was analyzed using Quantity One software version 4.4 (Bio-Rad Laboratories, Inc., Hercules, CA, USA) and normalized against that of $\beta$-actin.

Statistical analysis. The data are presented as the mean \pm standard deviation. Statistical analysis was performed by analysis of variance or two-tail Student's t-test using SPSS version 14 (SPSS, Inc., Chicago, IL, USA). P<0.05 was considered to indicate a statistically significant difference.

\section{Results}

Expression of miR-214 was increased in human OS tissues and cell lines. In order to determine the expression levels of miR-214 in OS tissues, RT-qPCR detection was performed in 22 pairs of OS tissues and normal tissues. The data demonstrated that miR-214 was significantly increased in the OS tissues compared with the matched normal tissues (Fig. 1A). Additionally, the expression of miR-214 in three OS cell lines, U2OS, Saos-2 and MG-63, was markedly increased compared with that in the human hFOB 1.19 osteoblast cell line (Fig. 1B).

Inhibition of miR-214 suppresses OS cell proliferation and migration. To investigate the role of miR-214 in OS cell proliferation, MG-63 cells were transfected with the miR-214 inhibitor, anti-miR-214, or control inhibitor, anti-ctrl. 
RT-qPCR demonstrated that the expression of miR-214 in the cells transfected with anti-miR-214 was significantly decreased compared with the control group (Fig. 2A). In addition, cell viability of the cells, which were transfected with anti-miR-214 were also markedly reduced (Fig. 2B) between 48 and $72 \mathrm{~h}$ following transfection. Transfection of anti-miR-214 also clearly suppressed colony formation in the MG-63 cells (Fig. 2C).

To investigate the effect of miR-214 on the motility of OS cells, in vitro migration and invasion assays were performed. It was revealed that transfection of anti-miR-214 significantly suppressed the in vitro migration and invasion abilities of the MG-63 cells (Fig. 2D and E).

miR-214 directly targets PTEN in OS cells. TargetScan 6.2 software was used to search the potential target gene of miR-214. PTEN was predicted to be a target of miR-214 (Fig. 3A). A luciferase activity assay revealed that miR-214 significantly suppressed the activity of PTEN luciferase in the WT 3'-UTR, however, not the Mut 3'-UTR in HEK293 cells (Fig. 3B). In addition, overexpression of miR-214 significantly suppressed the protein expression of PTEN. The protein expression of pAKT was significantly increased and the total AKT protein remained identical, while inhibition of miR-214 exhibited the opposite effects (Fig. 3C and D).

miR-214 is negatively correlated with PTEN in OS tissues. The mRNA expression of PTEN in the $22 \mathrm{OS}$ and the corresponding normal tissues was measured. The results revealed that the mRNA expression of PTEN was significantly decreased in OS tissues compared with the corresponding normal tissues (Fig. 4A). In addition, 35 OS cases were investigated and revealed that those patients expressing low levels of miR-214 exhibited a longer survival time compared with those expressing a high level (Fig. 4B).

\section{Discussion}

Emerging studies have revealed that miRNAs are involved in the progression of various types of cancer, including OS, via the regulation of the expression of multiple target genes involved in cancer progression and metastasis. Therefore, identification of specific miRNAs and their targets involved in tumorigenesis may provide valuable insight for the diagnosis and therapy of patients with human malignancies. The present study demonstrated that the expression of miR-214 was upregulated in OS tissues. Forced overexpression of miR-214 enhanced cell proliferation in MG-63 and U2OS cells, while miR-214 inhibition by its antisense oligonucleotides repressed cell proliferation. Therefore, the present study demonstrated that miR-214 may be an onco-microRNA in the progression of OS. However, further studies are required to elucidate the roles in vivo.

In addition, at the molecular level, these results revealed that PTEN is a direct target of miR-214 in OS cells. PTEN was originally identified as the tumor suppressor gene frequently lost on chromosome 10q23. The relevance of PTEN in cancer was addressed through the generation of germline knockout PTEN mice in several previous studies (31). These previous studies revealed the requirement of PTEN for embryonic development. Notably, heterozygous loss of this tumor suppressor in the mouse resulted in the development of cancer of multiple origins, as well as a lethal lymphoproliferative disease. In humans, germline loss and mutation of PTEN is observed in a group of autosomal dominant syndromes, PTEN hamartoma tumor syndromes, which are characterized by neurological disorders, multiple hamartomas and cancer susceptibility. Upregulation of PTEN can increase the expression of Caspase 3 to cause dysregulation of apoptosis in tumor cells, which forms the molecular mechanisms of PTEN contribution to tumorigenesis and progression of malignant tumors (32).

In conclusion, the present study described a novel miR-214/PTEN association. The data also provided a mechanism for PTEN dysregulation and its contribution to OS cell growth, migration and invasion. These results suggested that miR-214 may act as an oncogene in OS and represents a potential molecular target for OS therapy.

\section{References}

1. Klein MJ and Siegal GP: Osteosarcoma: Anatomic and histologic variants. Am J Clin Pathol 125: 555-581, 2006.

2. Tan ML, Choong PF and Dass CR: Osteosarcoma: Conventional treatment vs. gene therapy. Cancer Biol Ther 8: 106-117, 2009.

3. Yan K, Gao J, Yang T, Ma Q, Qiu X, Fan Q and Ma B: MicroRNA-34a inhibits the proliferation and metastasis of osteosarcoma cells both in vitro and in vivo. PLoS One 7: e33778, 2012

4. Amankwah EK, Conley AP and Reed DR: Epidemiology and therapies for metastatic sarcoma. Clin Epidemiol 5: 147-162, 2013.

5. Rainusso N, Wang LL and Yustein JT: The adolescent and young adult with cancer: State of the art - bone tumors. Curr Oncol Rep 15: 296-307, 2013.

6. Ameres SL and Zamore PD: Diversifying microRNA sequence and function. Nat Rev Mol Cell Biol 14: 475-488, 2013.

7. Sun K and Lai EC: Adult-specific functions of animal microRNAs. Nat Rev Genet 14: 535-548, 2013.

8. Kasinski AL and Slack FJ: Epigenetics and genetics. MicroRNAs en route to the clinic: Progress in validating and targeting microRNAs for cancer therapy. Nat Rev Cancer 11: 849-864, 2011.

9. Liang W, Gao B, Fu P, Xu S, Qian Y and Fu Q: The miRNAs in the pathogenesis of osteosarcoma. Front Biosci (Landmark Ed) 18: 788-794, 2013

10. Ziyan W, Shuhua Y, Xiufang W and Xiaoyun L: MicroRNA-21 is involved in osteosarcoma cell invasion and migration. Med Oncol 28: 1469-1474, 2011.

11. He C, Xiong J, Xu X, Lu W, Liu L, Xiao D and Wang D: Functional elucidation of MiR-34 in osteosarcoma cells and primary tumor samples. Biochem Biophys Res Commun 388: 35-40, 2009.

12. Song B, Wang Y, Xi Y, Kudo K, Bruheim S, Botchkina GI, Gavin E, Wan Y, Formentini A, Kornmann M, et al: Mechanism of chemoresistance mediated by miR-140 in human osteosarcoma and colon cancer cells. Oncogene 28: 4065-4074, 2009.

13. Zhao G, Cai C, Yang T, Qiu X, Liao B, Li W, Ji Z, Zhao J, Zhao H, Guo M, et al: MicroRNA-221 induces cell survival and cisplatin resistance through PI3K/Akt pathway in human osteosarcoma. PLoS One 8: e53906, 2013.

14. Li G, Cai M, Fu D, Chen K, Sun M, Cai Z and Cheng B. Heat shock protein 90B1 plays an oncogenic role and is a target of microRNA-223 in human osteosarcoma. Cell Physiol Biochem 30: 1481-1490, 2012.

15. Mao JH, Zhou RP, Peng AF, Liu ZL, Huang SH, Long XH and Shu Y: microRNA-195 suppresses osteosarcoma cell invasion and migration in vitro by targeting FASN. Oncol Lett 4: 1125-1129, 2012.

16. Cai CK, Zhao GY, Tian LY, Liu L, Yan K, Ma YL, Ji ZW, Li XX, Han K, Gao J, et al: miR-15a and miR-16-1 downregulate CCND1 and induce apoptosis and cell cycle arrest in osteosarcoma. Oncol Rep 28: 1764-1770, 2012.

17. Huang G, Nishimoto K, Zhou Z, Hughes D and Kleinerman ES: miR-20a encoded by the miR-17-92 cluster increases the metastatic potential of osteosarcoma cells by regulating Fas expression. Cancer Res 72: 908-916, 2012. 
18. MicroRNA-214 suppresses osteoblast differentiation by binding to Osterix. Bonekey Rep 2: 393, 2013.

19. Chen YF, Dong Z, Xia Y, Tang J, Peng L, Wang S and Lai D Nucleoside analog inhibits microRNA-214 through targeting heat-shock factor 1 in human epithelial ovarian cancer. Cancer Sci 104: 1683-1689, 2013.

20. Dong X, Liu H, Chen F, Li D and Zhao Y: MiR-214 promotes the alcohol-induced oxidative stress via down-regulation of glutathione reductase and cytochrome P450 oxidoreductase in liver cells. Alcohol Clin Exp Res 38: 68-77, 2014.

21. Yang T, Zhang GF, Chen XF, Gu HH, Fu SZ, Xu HF, Feng Q and Ni YM: MicroRNA-214 provokes cardiac hypertrophy via repression of EZH2. Biochem Biophys Res Commun 436: 578-584, 2013.

22. Shih TC, Tien YJ, Wen CJ, Yeh TS, Yu MC, Huang CH, Lee YS, Yen TC and Hsieh SY: MicroRNA-214 downregulation contributes to tumor angiogenesis by inducing secretion of the hepatoma-derived growth factor in human hepatoma. J Hepatol 57: 584-591, 2012.

23. Wang YS, Wang YH, Xia HP, Zhou SW, Schmid-Bindert G and Zhou CC: MicroRNA-214 regulates the acquired resistance to gefitinib via the PTEN/AKT pathway in EGFR-mutant cell lines. Asian Pac J Cancer Prev 13: 255-260, 2012.

24. Liu J, Luo XJ, Xiong AW, Zhang ZD, Yue S, Zhu MS and Cheng SY: MicroRNA-214 promotes myogenic differentiation by facilitating exit from mitosis via down-regulation of proto-oncogene N-ras. J Biol Chem 285: 26599-26607, 2010.
25. Zhang ZC, Li YY, Wang HY, Fu S, Wang XP, Zeng MS, Zeng YX and Shao JY: Knockdown of miR-214 promotes apoptosis and inhibits cell proliferation in nasopharyngeal carcinoma. PLoS One 9: e86149, 2014.

26. Yang TS, Yang XH, Wang XD, Wang YL, Zhou B and Song ZS: MiR-214 regulate gastric cancer cell proliferation, migration and invasion by targeting PTEN. Cell Int 13: 68, 2013.

27. Peng RQ, Wan HY, Li HF, Liu M, Li $X$ and Tang $H$ MicroRNA-214 suppresses growth and invasiveness of cervical cancer cells by targeting UDP-N-acetyl- $\alpha$-D-galactosamine:pol ypeptide $\mathrm{N}$-acetylgalactosaminyltransferase 7. J Biol Chem 287: 14301-14309, 2012.

28. Bubner B and Baldwin IT: Use of real-time PCR for determining copy number and zygosity in transgenic plants. Plant Cell Rep 23: 263-271, 2004

29. Li H and Yang BB: Stress response of glioblastoma cells mediated by miR-17-5p targeting PTEN and the passenger strand miR-17-3p targeting MDM2. Oncotarget 3: 1653-1668, 2012.

30. Han SB, Shin YJ, Hyon JY and Wee WR: Cytotoxicity of voriconazole on cultured human corneal endothelial cells. Antimicrob Agents Chemother 55: 4519-4523, 2011.

31. Aguissa-Touré AH and Li G: Genetic alterations of PTEN in human melanoma. Cell Mol Life Sci: 69: 1475-1491, 2012.

32. Zheng HC, Li YL, Sun JM, Yang XF, Li XH, Jiang WG, Zhang YC and Xin Y: Growth, invasion, metastasis, differentiation, angiogenesis and apoptosis of gastric cancer regulated by expression of PTEN encoding products. World J Gastroenterol: 9: 1662-1666, 2003. 